\title{
Knockdown of Lncrna PVT1 Enhances Radiosensitivity in Non-Small Cell Lung Cancer by Sponging Mir-195
}

\author{
Dapeng $\mathrm{Wu}^{\mathrm{a}}$ Yong $\mathrm{Li}^{\mathrm{b}}$ Huixiang Zhang ${ }^{\mathrm{a}}$ Xigang $\mathrm{Hu}^{\mathrm{a}}$ \\ aDepartment of Radiotherapy, ${ }^{b}$ Department of Cardiothoracic Surgery, Huaihe Hospital of Henan \\ University, Kaifeng, China
}

\section{Key Words}

Lncrna $・$ PVT1 $・$ MiR-195 • Radiosensitivity $・$ NSCLC

\begin{abstract}
Background/Aims: Plasmacytoma variant translocation 1 (PVT1) exerts an oncogenic role in many tumors, including lung cancer. However, the roles of PVT1 in regulating radiosensitivity of NSCLC and its underlying mechanism are still unclear. Methods: Expression levels of PVT1 and miR-195 in NSCLC tissues and cells were examined by qRT-PCR. Effects of PVT1 and miR-195 on cell proliferation, apoptosis and colony formation abilities were assessed by MTT assay, flow cytometry and colony formation assay. Luciferase reporter assay was performed to confirm the relationship between PVT1 and miR-195. Tumor xenograft experiments were conducted to observe the effect of PVT1 on radiosensitivity of NSCLC in vivo. Results: PVT1 was negatively correlated with miR-195 expression in NSCLC tissues and associated with poor prognosis of NSCLC patients. Expression of PVT1 and miR-195 varied inversely after irradiation in NSCLC cells. PVT1 knockdown or miR-195 overexpression enhanced radiosensitivity of NSCLC in vitro by inhibiting proliferation and inducing apoptosis. PVT1 directly interacted with miR-195 and regulated its expression. Moreover, PVT1 knockdown improved radiosensitivity of NSCLC cells in vitro and in vivo by sponging miR-195. Conclusion: Knockdown of PVT1 enhances radiosensitivity of NSCLC by sponging miR-195, providing a novel therapeutic target to improve radiotherapy efficiency in NSCLC.

\section{Introduction}

Lung cancer is the second common malignancy with the highest incidence in males and the second highest incidence in females, and is also the primary cause of cancer-associated death with 226, 000 new cases in the United States in 2012 [1,2]. Non-small cell lung cancer (NSCLC) is the largest subgroup and accounts for approximately $85 \%$ in all lung cancers [3].

D. Wu and Y. Li are contributed equally to this work.

\begin{tabular}{ll}
\hline Dapeng Wu & Department of Radiotherapy, Huaihe Hospital of Henan University, \\
No.8 Baobei Road, Gulou District, Kaifeng (China)
\end{tabular}


Most of NSCLC patients are diagnosed at an advanced stage, thus leading to a high mortality of NSCLC [4, 5]. High-energy radiotherapy (RT) and thoracic RT is currently the main nonsurgical approach for advanced NSCLC patients. However, the efficacy of RT is limited due to acquired radioresistance in the treatment of tumor [6]. The prognosis of NSCLC still remains poor owing to tumor recurrence and metastasis [7]. Therefore, identifying novel therapeutic targets to mitigate radioresistance is of great importance for improving the outcome of NSCLC patients.

Long non-coding RNAs (lncRNAs) are highly conserved transcripts consisting of more than 200 nucleotides in length with no protein-coding potential [8]. It is well established that lncRNAs play critical roles in many cellular biological processes, such as cell proliferation, apoptosis, as well as cancer progression [9]. Recent evidences have shown that lncRNA are generally dysregulated in various types of cancers and involved in tumorigenesis and development of cancers, including NSCLC $[10,11]$. For example, IncRNA X inactivatespecific transcript (XIST) was up-regulated and identified as an oncogene by epigenetically suppressing KLF2 expression in NSCLC [12]. High expression of IncRNA nuclear enriched abundant transcript1 (NEAT1) accelerated NSCLC cell growth and metastasis in vitro and in vivo through regulating miR-337-3p-E2F3 pathway [13]. Upregulation of tumor suppressor candidate 7 (TUSC7) repressed cell proliferation in NSCLC [14]. Plasmacytoma variant translocation 1 (PVT1) is located at chromosome8q24, a region shared with well known oncogene $c$-myc in various solid tumors, and exerts an carcinogenic effect in many tumors, such as lung cancer [15], gastric cancer [16] and pancreatic cancer [17]. Importantly, recent studies have demonstrated that PVT1 expression is significantly correlated with clinical parameters of numerous cancers, such as relapse and survival $[18,19]$. However, the role of PVT1 and its potential mechanism involved in modulating the radiosensitivity of NSCLC has never been investigated.

microRNAs (miRNAs), with 18-25 nucleotides in length, negatively regulate gene expression by targeting mRNA for some degree of degradation and/or suppression. Aberrant expression of miRNAs has been demonstrated to be closely associated with cancer development and progression, and play pivotal roles in diverse cellular progresses, such as cell differentiation, stress response, proliferation and apoptosis [20]. Currently, accumulating evidence showed that miRNAs could influence the cell response to radiation and regulate cell radiosensitivity of NSCLC [21]. miR-195 was reported to act as a tumor suppressor in NSCLC by modulating different target genes [22-24]. However, the role of miR-195 in regulating radiosensitivity of NSCLC is still unknown.

In the present study, the effects of PVT1 on radiosensitivity of NSCLC cells were first evaluated. Furthermore, the underlying mechanism of PVT1 in regulating radiosensitivity of NSCLC was also investigated.

\section{Materials and Methods}

Patients and tumor samples

Thirty-one pairs of NSCLC tissues

(20 adenocarcinoma and 11 squamous cell carcinoma) and adjacent normal lung tissues were obtained from Huaihe Hospital of Henan University. All patients with histopathologically confirmed NSCLC had never subjected to preoperative chemo- or radio-therapy. The clinicopathological characteristics of the NSCLC patients are summarized in

Table 1. Correlation between PVT1 expression and clinicopathological features in NSCLC patients, ${ }^{*} \mathrm{P}<0.05$ was considered significantly significant

\begin{tabular}{|c|c|c|c|c|c|}
\hline \multirow{2}{*}{ Characteristics } & \multirow{2}{*}{ Group } & \multirow{2}{*}{$\begin{array}{l}\text { Total } \\
(\mathrm{n}=31)\end{array}$} & \multicolumn{2}{|c|}{ PVT1 expression } & \multirow{2}{*}{$P$ value } \\
\hline & & & High $(n=15)$ & Low $(n=16)$ & \\
\hline \multirow[t]{2}{*}{ Gender } & Male & 22 & 10 & 12 & 0.704 \\
\hline & Female & 9 & 5 & 4 & \\
\hline \multirow[t]{2}{*}{ Age (years) } & $<60$ & 14 & 8 & 6 & 0.376 \\
\hline & $\geq 60$ & 17 & 7 & 10 & \\
\hline \multirow[t]{2}{*}{ Smoking } & No & 12 & 7 & 5 & 0.379 \\
\hline & Yes & 19 & 8 & 11 & \\
\hline \multirow[t]{2}{*}{ TNM stage } & $\mathrm{I} / \mathrm{II}$ & 13 & 3 & 10 & $0.017^{*}$ \\
\hline & III/IV & 18 & 12 & 6 & \\
\hline \multirow[t]{2}{*}{ Lymph nodes metastasis } & NO & 19 & 6 & 13 & $0.018^{*}$ \\
\hline & YES & 12 & 9 & 3 & \\
\hline
\end{tabular}




\section{Cellular Physiology Cell Physiol Biochem 2017;42:2453-2466 \begin{tabular}{l|l|l} 
and Biochemistry 10.1159/000480209 & $\begin{array}{l}\text { C) } 2017 \text { The Author(s). Published by S. Karger AG, Basel } \\
\text { www.karger.com/cpb }\end{array}$ \\
\hline
\end{tabular}}

Wu et al.: Knockdown of PVT1 Enhances Radiosensitivity in NSCLC

Table 1. All specimens were collected during tumor resection and immediately stored in liquid nitrogen until analysis. To observe the expression changes of PVT1 and miR-195 before and after radiation therapy, another 11 patients with biopsy-confirmed diagnosis of NSCLC were enrolled our study. Written informed consents were provided by all participants following protocols. The experiments were conducted with approval of the Ethics Review Committee of Huaihe Hospital.

\section{Cell lines and cell culture}

Human lung cell lines (A549, H157, HCC827 and H838) and normal bronchial epithelial cell line 16HBE were obtained from the American Type Culture Collection (ATCC, Manassas, VA, USA). All cell lines were maintained in Dulbecco's modified Eagle'smedium (DMEM) medium (Hyclone, Logan, UT, USA) supplemented with 10\% heat-inactivated fetal bovine serum (FBS; Invitrogen, Grand Island, NY, USA), 100 $\mathrm{U} / \mathrm{ml}$ penicillin, and $100 \mu \mathrm{g} / \mathrm{ml}$ streptomycinin a humidified incubator containing $5 \% \mathrm{CO}_{2}$ at $37^{\circ} \mathrm{C}$. The culture medium was replaced every 3-4 days.

\section{Cell transfection}

miRNA-195 mimics (miR-195), miRNA negative control (miR-NC), miRNA-195 inhibitors, siRNAs specifically targeting PVT1 (si-PVT1\#1, si-PVT1\#2 and si-PVT1\#3), siRNA negative control (si-NC) were chemically synthesized by GenePharma Co. Ltd. (Shanghai,China). The cDNA of PVT1 was amplified and subcloned into the Kpn I and BamH I sites of pcDNA (Invitrogen) and named as pcDNA-PVT1. The pcDNA empty vector (pcDNA-NC) was used as the control. A549 and H157 cells in logarithmic phase were seeded into 6-well plates at a final concentration of $4 \times 10^{5} /$ well and cultured for $24 \mathrm{~h}$ before transfection. Cell transfection with different siRNAs, pcDNAs or miRNAs was performed using Lipofectamine 2000 (Invitrogen). Further experiments were carried out at indicated times after transfection.

\section{Ionizing radiation treatment of cells}

Transfected cells $\left(5 \times 10^{4}\right)$ were irradiated by a linear accelerator (Varian Medical Systems, USA) in 25-T flasks. Cells were exposed to different doses of irradiation treatment (0, 2, 4, 6, and 8 Gy) with a 6-MV photon beam at a dose rate of $3.5 \mathrm{~Gy} / \mathrm{min}$ and $160 \mathrm{kv}$ X-ray energy. After 24-96 h, cells were used for other analysis. For time-course experiment, cells received 4 Gy X-ray and then were collected for qRT-PCR analysis every $2 \mathrm{~h}$ within $24 \mathrm{~h}$ after irradiation.

\section{Quantitative real-time PCR ( $q$ RT-PCR)}

Total RNA from the collected tissues and cultured cells was extracted by using TRIZOL regent (Invitrogen). $1 \mu \mathrm{g}$ of total RNA was reversely transcribed to complementary DNA (cDNA) using SuperScript First StrandcDNA System (Invitrogen). Then PCR analyses of PVT1 and miR-195 were carried out with a LightCycler Brilliant SYBR GreenRT-qPCR kit (Roche Applied Science, Indianapolis, IN, USA) and TaqMan MicroRNA Assay Kit (Applied Biosystems, Foster City, CA, USA) on an ABI Prism 7500 real-time PCR system (Applied Biosystems), respectively. The relative expressions of PVT1 and miR-195 were calculated using $2^{-\triangle \triangle C T}$ method and normalized to GAPDH or U6 snRNA. The primers used were as follows: PVT1: 5'-TGAGAACTGTCCTTACGTGACC-3' (forward) and 5'-AGAGCACCAAGACTGGCTCT-3' (reverse); GAPDH: 5'-GGAGCGAGATCCCTCCAAAAT-3' (forward) and 5'-GGCTGTTGTCATACTTCTCATGG-3' (reverse); miR195, CGTAGCAGCACAGAAAT (forward) and GTGCAGGGTCCGAGGT (reverse); U6, CTCGCTTCGGCAGCACA (forward) and AACGCTTCACGAATTTGCGT (reverse). The PCR procedures were performed as follows: $95^{\circ} \mathrm{C}$ for $2 \mathrm{~min}$, followed by $95^{\circ} \mathrm{C}$ for $30 \mathrm{~s}, 60^{\circ} \mathrm{C}$ for $30 \mathrm{~s}$ and 30 cycles of $72^{\circ} \mathrm{C}$ for $1 \mathrm{~min}$, and $72^{\circ} \mathrm{C}$ for $10 \mathrm{~min}$. PCR conditions of miR- 195 were $95^{\circ} \mathrm{C}$ for $10 \mathrm{~min}, 40$ cycles of $95^{\circ} \mathrm{C}$ for $15 \mathrm{~s}$, and $60^{\circ} \mathrm{C}$ for $60 \mathrm{~s}$.

Western blot analysis

Western blot analysis was performed with antibody against Ser139-phosphorylated H2AX $(\gamma-\mathrm{H} 2 \mathrm{AX})$ (Abcom, Cambridge, MA, USA) as described previously [9]. Anti- $\beta$-actin monoclonal antibody (Santa Cruz Biotechnology, Santa Cruz, CA, USA) was used as an internal control.

Cell proliferation assay

Cells were inoculated into a 96-well plate at a density of $2 \times 10^{3} /$ well and transiently transfected with si-PVT1, miR-195, si-PVT1 + miR-195 inhibitor, or matched controls. After 24 h, cells were exposed 


\section{Cellular Physiology Cell Physiol Biochem 2017;42:2453-2466 \begin{tabular}{l|l|l} 
and Biochemistry 10.1159/000480209 & $\begin{array}{l}\text { D) } 2017 \text { The Author(s). Published by S. Karger AG, Basel } \\
\text { www.karger.com/cpb }\end{array}$
\end{tabular}}

Wu et al.: Knockdown of PVT1 Enhances Radiosensitivity in NSCLC

to 4 Gy dose X-ray. At 0, 24, 48, 72 and 96 h after irradiation, $20 \mu \mathrm{L}$ MTT solution ( $5 \mathrm{mg} / \mathrm{ml}$; Sigma Aldrich Company Ltd, Poole, Dorset, UK) was added to each well for another $4 \mathrm{~h}$ incubation at $37^{\circ} \mathrm{C}$. Subsequently, the medium was discarded and $150 \mu \mathrm{L}$ of dimethylsulfoxide (DMSO) was supplemented to dissolve the formazan products. The absorbance at $490 \mathrm{~nm}$ was measured $10 \mathrm{~min}$ later using a microplate reader (BioRad, Hercules, CA, USA). All experiments were repeated for at least three times and the average results were calculated.

\section{Luciferase activity assay}

PVT1 fragments containing the predicted wild type (wt) or mutant (mut) miR-195 binding sites were synthesized (RiboBio Co., Ltd., Guangzhou, China) and cloned into the XhoI and XbaI sites of the downstream of Renila luciferase gene in the vector pmirGLO (Promega, Madison, WI, USA). The recombinant plasmids were designated as pmirGLO-PVT1-wt and pmirGLO-PVT1-mut. For luciferase activity assay, approximately $1 \times 10^{4}$ cells were plated into 96-well plates and co-transfected with $0.5 \mu \mathrm{g}$ pmirGLO-PVT1-wt or pmirGLOPVT1-mut and $50 \mathrm{nM}$ miR-195 or miR-NC by Lipofectamine 2000 (Invitrogen). Firefly luciferase gene in the vector pmirGLO-control (Promega) was used as the endogenous control to detect transfection efficiency. Cells were harvested and lysed at $24 \mathrm{~h}$ after transfection and the luciferase activity was measured using the Dual-Luciferase Reporter Assay system (Promega). Firefly luciferase activity was normalized to the corresponding Renilla luciferase. The above experiments were performed in triplicate.

\section{Colony formation assay}

A predetermined number of cells $\left(1 \times 10^{3}\right)$ were seeded in six-well culture plates with complete medium and transfected with si-PVT1, miR-195, si-PVT1 + miR-195 inhibitor or matched controls. Following by 24 $\mathrm{h}$ incubation, the cells were treated with indicated doses of $\mathrm{X}$ irradiation $(0,2,4,6$, and 8 Gy) at a dose rate of $3.5 \mathrm{~Gy} / \mathrm{min}$ and seeded into $60 \mathrm{~mm}$ dishes for 14 days incubation at $37^{\circ} \mathrm{C}$. Then cells were washed twice with PBS, fixed with methanol for $5 \mathrm{~min}$ and stained with crystal violet for $3 \mathrm{~min}$ at $37^{\circ} \mathrm{C}$. The number of colonies with more than 50 cells was counted by microscopic inspection. The plating efficiency (PE) was computed using the following formula: $\mathrm{PE}=$ number of colonies $/$ number of cells seeded $\times 100 \%$. The survival fractions (SF) were calculated by the following equation: $\mathrm{SF}=$ number of colonies $/$ number of cells seeded $\times(1 / \mathrm{PE})$. Three independent experiments were repeated.

Flow cytometric analysis of apoptosis

Cells $\left(1 \times 10^{6}\right.$ cells /well $)$ were seeded in six-well plates and transfected with si-PVT1, miR-195, si-PVT1 + miR-195 inhibitor or matched controls. 24 h post transfection, cells were treated with a 4 Gy irradiation. The cells were collected $24 \mathrm{~h}$ after irradiation treatment and the percentage of apoptotic cells was examined by a FACScalibur Flow Cytometry (BD Biosciences, San Jose, CA, USA) using annexin-V fluorescein isothiocyanate (FITC) and propidium iodide (PI) apoptosis detection kit (Invitrogen). Each experiment was conducted at least three times.

\section{Tumor xenograft experiments}

All procedures involving mice in this study were approved by Institutional Research Committee of Huaihe Hospital of Henan University. All BALB/c nude mice aged 6-7 weeks and weighing 22-24 g (GuangDong Experimental Animal Centre) were divided into three groups (5 mice/group) and kept in an aseptic, constant temperature and humidity condition. Tumors were generated by subcutaneously injection into the right flank of nude mice with $1 \times 10^{6}$ stably transfected A549 cells resuspended in $100 \mu \mathrm{L}$ serum-free medium. The lentiviral particles with PVT1 hairpin oligonucleotides (sh-PVT1) or negative control (sh-NC) and lentiviral miR-195 inhibitor expression plasmids (miR-195 inhibitor) were purchased from GenePharma (Shanghai, China). When the xenograft tumor volumes reached an average volume of approximately 300 $\mathrm{mm}^{3}$, mice were irradiated with 4 Gy X-rays once a week. Tumor volumes and mice body weight were regularly determined every 3 days for 21 days after irradiation. The tumor volume was calculated by the formula: $1 / 2 \times$ length $\times$ width $^{2}$. At 21 days after the first irradiation, the mice were sacrificed and the tumors were excised, immediately imaged and weighted.

Statistical analysis

Student's $t$ test and One-way Analysis of Variance (ANOVA) were used to determine statistical differences. The results of the quantitative data were presented as the mean \pm standard deviation (SD) of 
three independent experiments and analyzed using the GraphPad Prism version 5.0 program (GraphPad Software, CA, USA). A P-Value less than 0.05 was considered statistically significant.

\section{Results}

PVT1 is negatively correlated with miR-195 expression and associated with poor prognosis in NSCLC tissues

To verify the biological functions of PVT1 and miR-195 in NSCLC development, qRTPCR was performed to analyze the expressions of PVT1 and miR-195 in 31 pairs of NSCLC tissues and adjacent normal lung tissues. As shown in Fig. 1A, PVT1 expression was significantly higher in NSCLC tissues than that in adjacent normal tissues. miR-195 expression was dramatically downregulated in NSCLC tissues compared with corresponding normal tissues (Fig. 1B). To observe the expression changes of PVT1 and miR195 before and after radiation therapy, another 11 patients with biopsy-confirmed diagnosis of NSCLC were enrolled our study. qRT-PCR analysis demonstrated that PVT1 expression was increased (Fig. 1C) while miR-195 expression was decreased (Fig. 1D) after radiation treatment in NSCLC tissues. Moreover, a significantly negative correlation between PVT1 and miR-195 was observed in NSCLC tissues (Fig. 1E). In addition, the Kaplan-Meier plots presented that NSCLC patients with high expression of PVT1 had a poor prognosis compared with patients with low expression of PVT1 (Fig. 1F). Furthermore, statistical analysis of clinical relevance of PVT1 expression in NSCLC demonstrated that that high expression of PVT1 in NSCLC was significantly correlated with TNM stage $(P=0.017)$ and lymph node metastasis $(P=0.018)$, as presented in Table 1. These data suggested that PVT1 and miR-195 may be involved in the development of radiation resistance in NSCLC progression.

Expression levels of PVT1 and miR-195 vary inversely under irradiation in NSCLC cells

The expression levels of PVT1 and miR-195 in NSCLC cells were further investigated and qRT-PCR analyses showed that PVT1 was substantially upregulated in a panel of 4 NSCLC cells (A549, H838, H157, and HCC827) compared with that in normal bronchial epithelial cell line 16HBE, especially inA549 and H157 cells (Fig. 2A). Additionally, miR-195 was expressed at a lower level in A549, H838, H157, and HCC827 cells than that in 16HBE cells, especially in A549 
and H157 cells (Fig. 2B). To further explore whether radiation could affect the expression of PVT1 and miR-195 in NSCLC cells, A549 and H157 cells were treated with 4 Gy X-ray and the expression levels of PVT1 and miR-195 were evaluated every 2 h. The results indicated that the expression level of PVT1 was significantly increased in both A549 and H157 cells since the 10th hour after 4 Gy irradiation treatment (Fig. 2C and 2D). Conversely, miR-195 was remarkably downregulated since the 8th hour after 4 Gy irradiation treatment (Fig. 2E and 2F). The results suggested that there were inverse expression changes between PVT1 and miR195 in NSCLC cells under irradiation.

PVT1 knockdown improves radiosensitivity of NSCLC cells

Based on the upregulation of PVT1 in response to irradiation in A549 and H157 cells, we supposed that PVT1 could affect the radiosensitivity of NSCLC cells. SiRNA-mediated PVT1 knockdown was performed to further explore the role of PVT1 in radiosensitivity of NSCLC cells. The transfection efficiencies of si-PVT1\#1, si-PVT1\#2 and si-PVT1\#3 in A549 and H157 cells were confirmed by qRT-PCR. Among them, only Si-PVT1\#2 transfection led to approximately $70 \%$ knockdown of PVT1 in A549 (Fig. 3A) and H157 (Fig. 3B) cells. Thus, si-PVT1\#2 was chosen for subsequent experiments. A549 and H157 cells transfected with si-NC or si-PVT1 were exposed to 4 Gy irradiation treatment. The results implied that knockdown of PVT1 or radiation therapy alone inhibited proliferation of A549 and H157 compared to si-NC group. Combination of si-PVT1 and radiation therapy markedly inhibited proliferation of A549 and H157 cells compared with si-PVT1 or $4 \mathrm{~Gy}+$ si-NC group (Fig. 3C and 3D). Besides, knockdown of PVT1 or radiation therapy alone induced apoptosis of A549 and H157 cells. Combination of si-PVT1 and radiation therapy strikingly increased the apoptosis rates of A549 and H157 cells compared with si-PVT1 or 4 Gy + si-PVT1 group (Fig. 3E and 3F). Furthermore, the colony formation assay showed that PVT1 downregulation resulted in a significant decrease of colony survival fraction in both A549 (Fig. 3G) and H157 (Fig. 3H) cells. All the results suggested that PVT1 knockdown enhanced the sensitivity of NSCLC cells to ionizing radiation by suppressing cell survival and inducing cell apoptosis. 


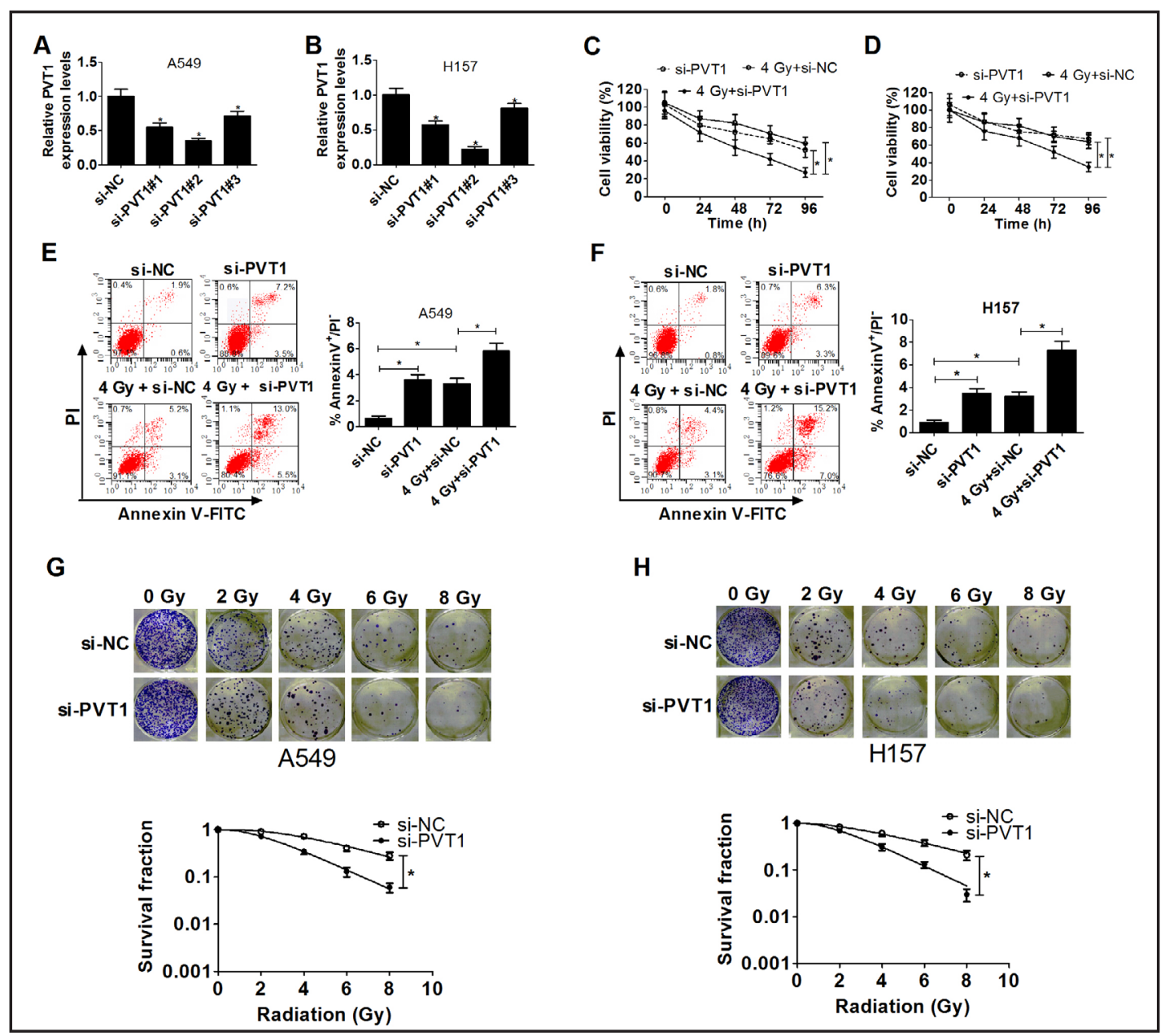

Fig. 3. Effect of PVT1 knockdown on radiosensitivity of NSCLC cells. The transfection efficiencies of siPVT1\#1, si-PVT1\#2 and si-PVT1\#3 were confirmed by qRT-PCR in A549 (A) and H157 (B) cells. Values are presented as mean $\pm \mathrm{SD}, \mathrm{n}=3$. MTT assay was performed to evaluate cell viability at $24 \mathrm{~h}, 48 \mathrm{~h}, 72 \mathrm{~h}$ and $96 \mathrm{~h}$ after A549 (C) and H157 (D) cells transfected with si-PVT1 or si-NC were exposed to 4 Gy irradiation. Cell viability was represented as a percentage of si-NC cells (non irradiated controls, 100\%). Values are presented as mean \pm SEM, $n=3$. Flow cytometry analyses were carried out to determine the apoptosis rate of A549 (E) and H157 (F) cells transfected with si-PVT1 or si-NC under 4 Gy irradiation. Apoptosis rate was determined by the fraction of Annexin-V binding cells/propidium iodide negative cells. Values are presented as mean $\pm \mathrm{SD}, \mathrm{n}=3$. Colony formation assay was used to assess colony survival rate 14 days after A549 (E) and H157 (F) cells transfected with si-PVT1 or si-NC were treated with different doses of irradiation. Values are presented as mean $\pm \mathrm{SD}, \mathrm{n}=3 .{ }^{*} \mathrm{P}<0.05$.

miR-195 overexpression increases radiosensitivity of NSCLC cells

To further explore the effect of miR-195 on radiosensitivity of NSCLC cells, miR-195- or miR-NC-transfected A549 and H157 cells were treated with 4 Gy X-ray. miR-195 expression was conspicuously improved in miR-195-transfected A549 (Fig. 4A) and H157 (Fig. 4B) cells compared with miR-NC group. MTT assay revealed that either miR-195 transfection or irradiation treatment exhibited a decreased cell proliferation rate compared si-NC group in A549 and H157 cells. Combination of miR-195 overexpression and radiation therapy markedly inhibited proliferation of A549 and H157 cells compared with miR-195 or 4 Gy + miR-NC group (Fig. 4C and 4D). In addition, A549 (Fig. 4E) and H157 (Fig. 4F) cells transfected with miR-195 or treated with 4 Gy irradiation both demonstrated a higher apoptosis rate than si-NC-transfected cells. Combination of miR-195 overexpression and radiation therapy 
Fig. 4. Effect of miR-195 upregulation on radiosensitivity of NSCLC cells. A549 and H157 cells were transfected with miR-195 or miR-NC. At $24 \mathrm{~h}$ after transfection, different irradiation treatment was conducted. miR-195 expression in transfected A549 (A) and H157 (B) cells were examined by qRT-PCR. Values are presented as mean $\pm \mathrm{SD}, \mathrm{n}=3$. Cell viability at 24 h, $48 \mathrm{~h}, 72 \mathrm{~h}$ and $96 \mathrm{~h}$ in transfected A549 (Fig. 4C) and H157 (Fig. 4D) cells were examined by MTT assay with 4 Gy irradiation exposure. Cell viability was represented as a percentage of miR-NC cells (non irradiated controls, 100\%). Values are presented

A

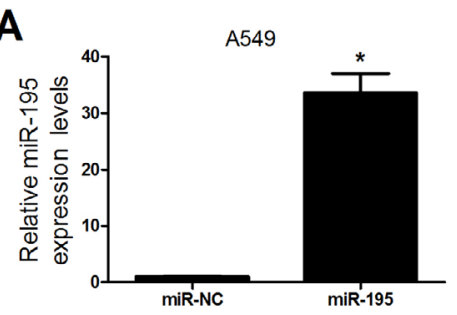

C

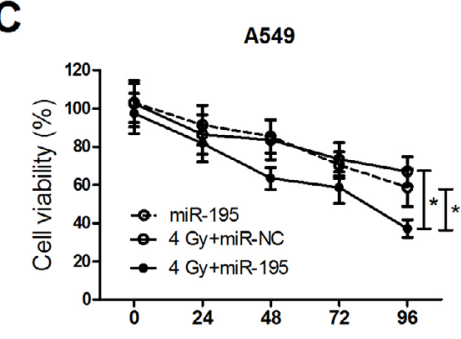

$\mathbf{E}$

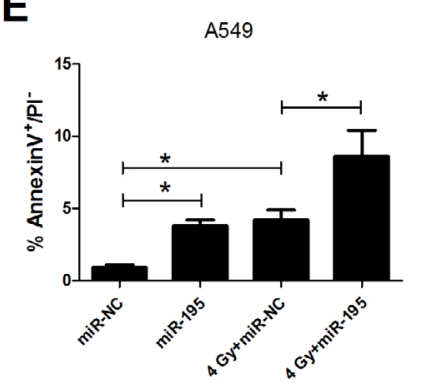

G

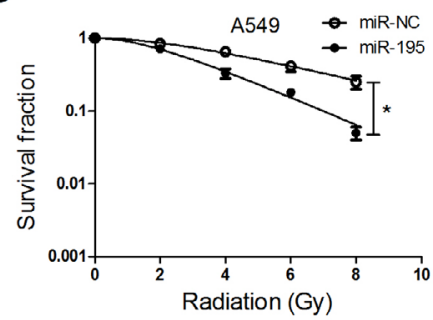

B

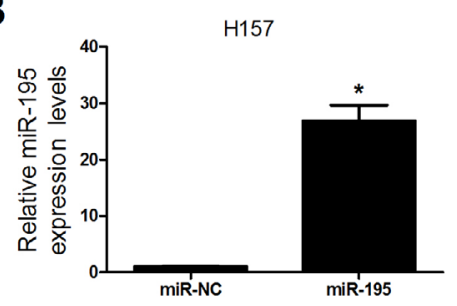

D

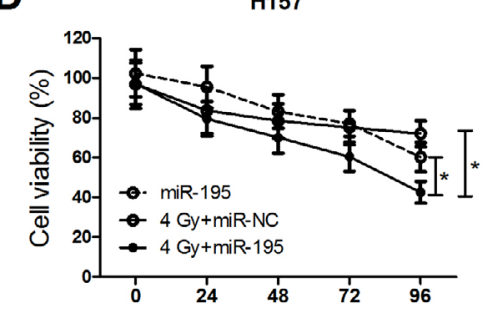

$\mathbf{F}$

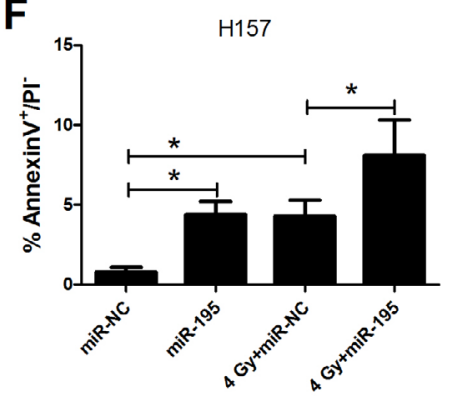

H

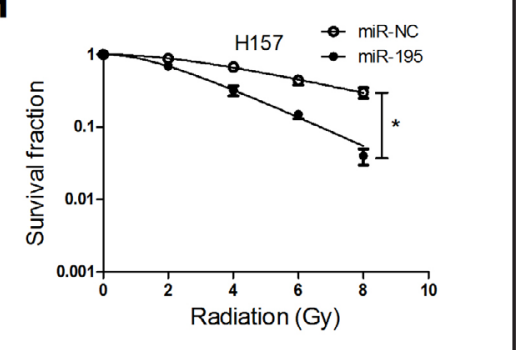
as mean \pm SEM, $\mathrm{n}$

= 3. Apoptosis of transfected A549 (Fig. 4E) and H157 (Fig. 4F) cells was assessed by flow cytometry under 4 Gy irradiation. Apoptotic rate was determined by the fraction of Annexin-V binding cells/propidium iodide negative cells. Values are presented as mean $\pm S D, n=3$. The colony survival fraction of transfected A549 (Fig. 4G) and H157 (Fig. 4H) cells was detected by colony formation assay with different doses of irradiation. Values are presented as mean $\pm \mathrm{SD}, \mathrm{n}=3 .{ }^{*} \mathrm{P}<0.05$.

markedly increased apoptosis rates of A549 and H157 cells compared with miR-195 or 4 Gy + miR-NC group (Fig. 4E and 4F). Besides, miR-195 overexpression obviously reduced colony formation rates in A549 (Fig. 4G) and H157 (Fig. 4H) cells compared with miR-NC group. These data revealed that miR-195 overexpression played a critical role in increasing radiosensitivity of NSCLC cells.

\section{PVT1 directly interacts with miR-195 and regulates its expression}

Previous studies have reported that IncRNA may function as a molecular sponge to competitively inhibit miRNA expression [25]. Through online bioinformatics database (starBase, v2.0, http://starbase.sysu.edu.cn/), PVT1 was predicted to contain a putative 
binding site of miR-195 (Fig. 5A). To further confirm whether miR-195 could directly bind to PVT1, we constructed the luciferase reporter plasmids containing the predicted miR-195 binding site (pmirGLO-PVT1-wt) or mutated miR-195 binding sequence (pmirGLO-PVT1mut) to co-transfect with miR195 or miR-NC into A549 and H157 cells. Luciferase reporter assay results showed that cotransfection with pmirGLOPVT1-wt and miR-195 resulted in a significant decrease of luciferase activities, whereas cotransfection of pmirGLOPVT1-mut and miR-195 had no obvious change on luciferase activities in A549 (Fig. 5B) and H157 (Fig. 5C) cells. To further verify the mutual effect between PVT1 and miR-195, qRT-PCR was performed to evaluate the expression of miR-195 in A549 and H157 cells transfected with si-PVT1, pcDNA-PVT1 or corresponding controls. The results indicated that PVT1 knockdown significantly improved the expression of miR-195 while PVT1 overexpression dramatically inhibited the expression of miR-195 in A549 (Fig. 5D) and H157 (Fig. 5E) cells. The results indicated that PVT1 could directly interact with miR195 and negatively regulate the expression of miR-195.

PVT1 regulates radiosensitivity of NSCLC cells by sponging miR-195 in vitro

Based on the regulatory role of PVT1 on miR-195 expression, we further investigated the effect of this axis on radiosensitivity of NSCLC cells. A549 and H157 cells transfected with si-NC or si-PVT1 or combined si-PVT1 and miR195 inhibitor were exposed to irradiation. MTT assay results suggested that PVT1 knockdown significantly decreased cell proliferation rates in vitro while miR-195 inhibitor completely reversed this effect in A549 (Fig. 6A) and H157 (Fig. 6B) cells. Besides, miR-195 inhibitor significantly recuperated si-PVT1induced cell apoptosis in A549 (Fig. 6C) and H157 (Fig. 6D) cells in vitro. Moreover, PVT1 downregulation significantly reduced colony formation rates in A549 (Fig. 6E) and H157 (Fig. 6F) cells in vitro, which was absolutely restored by miR-195 inhibitor. Taken together, the results revealed that the PVT1 knockdown improved radiosensitivity of NSCLC cells by sponging miR-195 in vitro.

PVT1 knockdown enhances radiosensitivity of NSCLC cells by sponging miR-195 in vivo

We further investigated whether PVT1 could regulate radiosensitivity of NSCLC cells in vivo by sponging miR-195. A549 cells stably transfected with sh-PVT1, sh-NC or sh-PVT1 


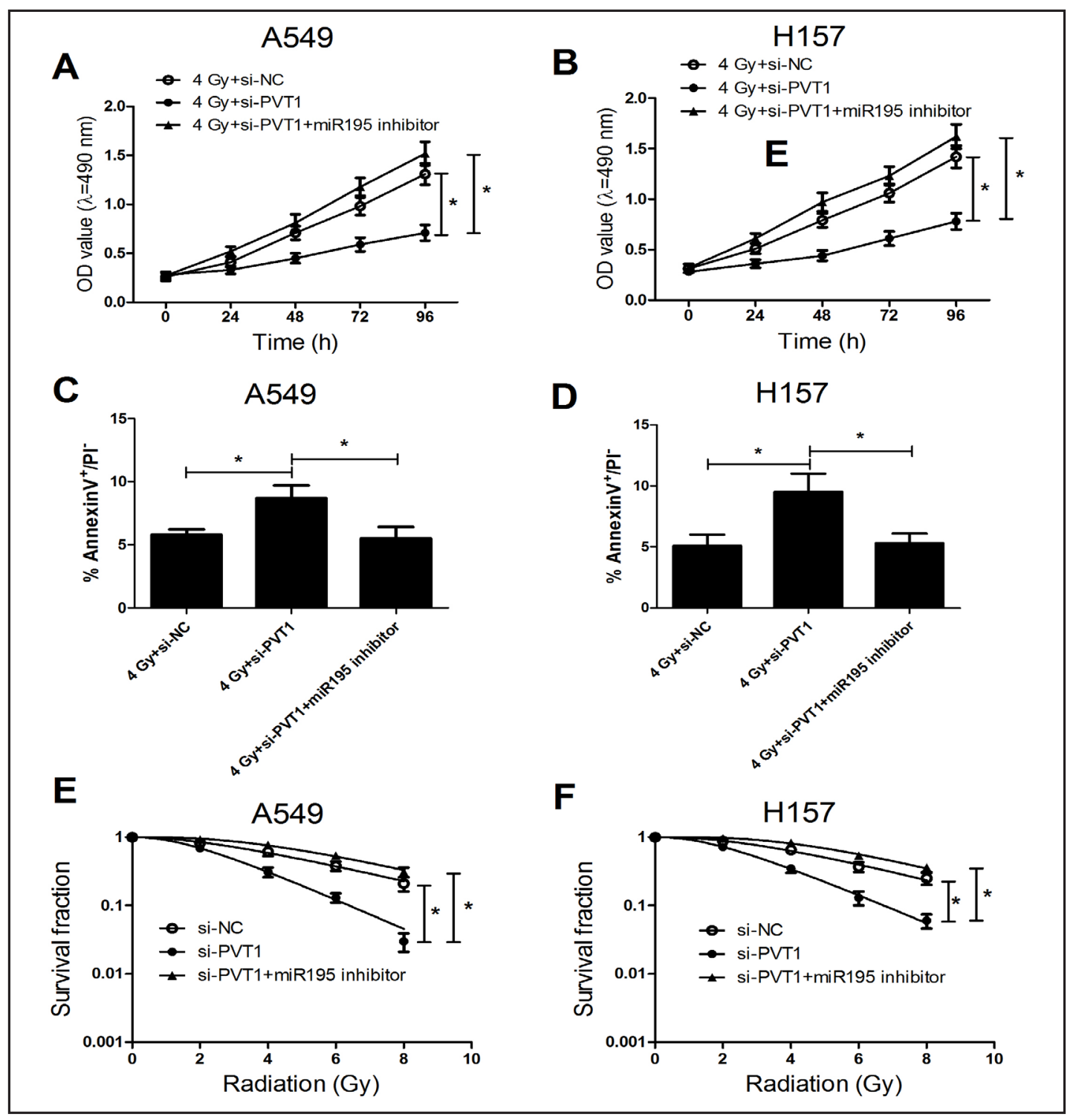

Fig. 6. miR-195 downregulation abated the effects of si-PVT1 on radiosensitivity of NSCLC cells in vitro. A549 and H157 cells were transfected with si-PVT1, si-NC or si-PVT1 + miR-195 inhibitor. At $24 \mathrm{~h}$ after transfection, different irradiation treatment was conducted. MTT assay was performed to assess cell viability in transfected A549 (A) and H157 (B) cells at 24 h, 48 h, 72 h and 96 h after 4 Gy irradiation. Values are presented as mean \pm SEM, $n=3$. Flow cytometry was carried out to evaluate apoptosis (Annexin V-positive/propidium iodide-negative) of transfected A549 (C) and H157 (D) cells under 4 Gy irradiation. Values are presented as mean $\pm S D, n=3$. The clonogenic survival curves of transfected A549 (E) and H157 (F) cells were established by colony formation assay after 4 Gy irradiation. Values are presented as mean \pm $\mathrm{SD}, \mathrm{n}=3 .{ }^{*} \mathrm{P}<0.05$.

+ miR-195 inhibitor were subcutaneously inoculated into nude mice, which were treated with X-ray once a week. As presented in Fig. 7A, PVT1 knockdown or combination of PVT1 knockdown and miR-195 inhibitor had no impact on mice body weight. In accordance with in vitro results, tumor volumes from the PVT1 knockdown group were obviously smaller than those from the control group at indicated times, whereas miR-195 inhibitor significantly relieved this effect (Fig. 7B). In addition, the tumor weight in PVT1 knockdown group was remarkably less than control group, which was completely overturned by miR-195 


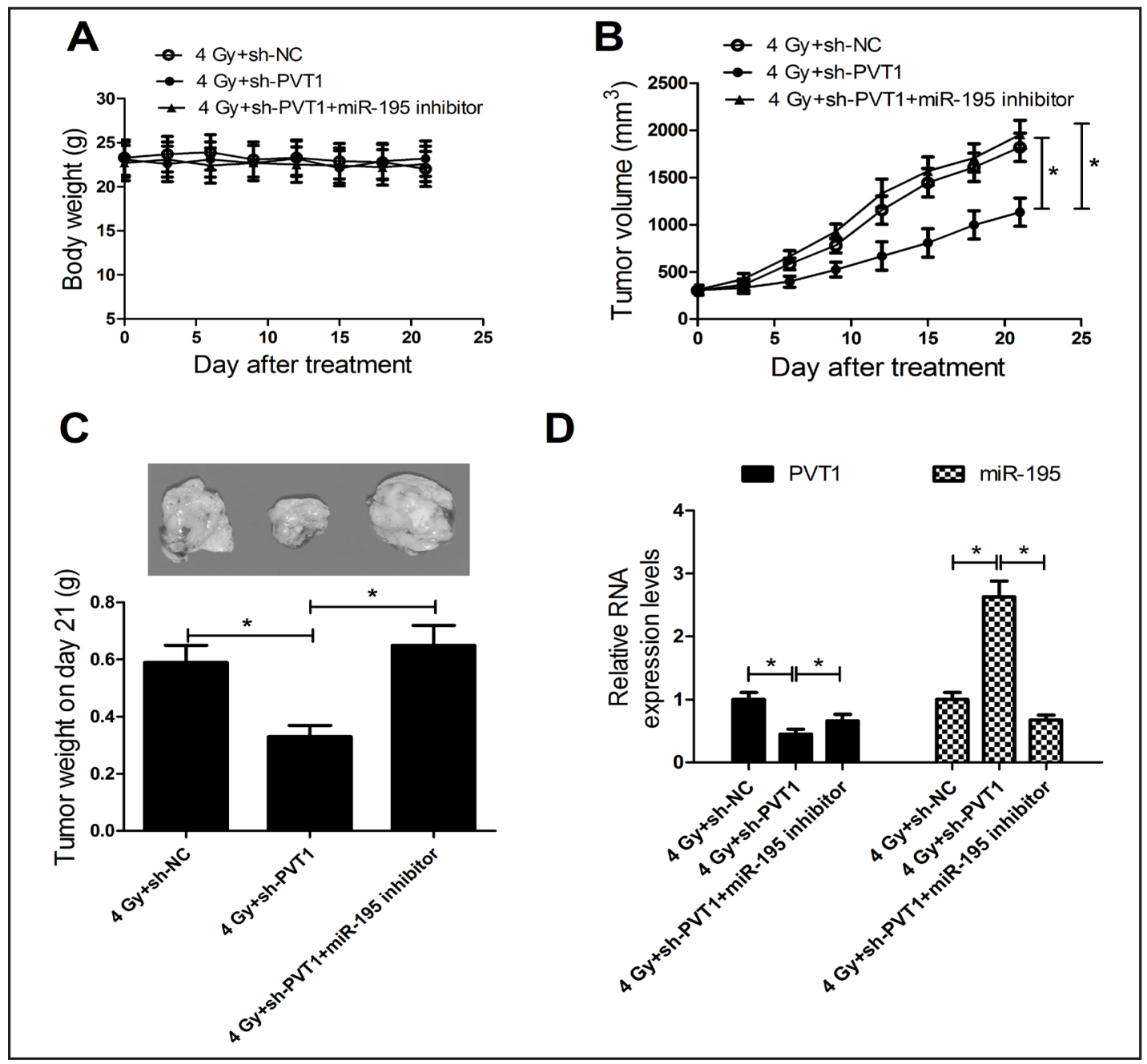

Fig. 7. Knockdown of PVT1 sensitized NSCLC cells to radiotherapy by sponging miR-195 in vivo. A549 cells stably transfected with sh-PVT1, sh-NC or sh-PVT1 + miR-195 inhibitor were subcutaneously inoculated into nude mice, which were treated with X-ray once a week. At 21 day after irradiation, the tumors were resected and weighted. Mice body weight (A) and tumor volumes (B) were measured every 3 days after xenograft tumor was irradiated with 4 Gy X-rays. (C) Tumors were harvested, immediately imaged and weighted at 21 days of irradiation. (D) Expressions of PVT1 and miR-195 in resected tumors were evaluated by qRT-PCR. All values are presented as mean $\pm \mathrm{SD}, \mathrm{n}=3 .{ }^{*} \mathrm{P}<0.05$.

inhibitor (Fig. 7C). The expressions of PVT1 and miR-195 in resected tumors were verified by qRT-PCR. As illustrated in Fig. 7D, tumors derived from sh-PVT1-transfected NSCLC cells exhibited downregulated PVT1 and upregulated miR-195, whereas miR-195 inhibitor markedly overturned these effects. These results indicated PVT1 knockdown enhanced radiosensitivity of NSCLC cells in vivo by sponging miR-195.

\section{Discussion}

Modern radiotherapy is generally considered one of the most effective treatments for inoperable cancer, including NSCLC at advanced stages [26]. Nevertheless, radioresistance induced by intrinsic mechanism and extracellular factors through intercellular communications is the main obstacle limiting the treatment efficacy of radiotherapy for

\section{KARGER}


NSCLC, followed by distant metastasis and secondary lung cancer [27]. Therefore, a profound understanding of the molecular mechanism underlying the development of radiation resistance in NSCLC may contribute to improving the therapeutic effects and prognosis of NSCLC patients.

Up to now, a growing number of miRNAs have been identified to be important regulators of radiosensitivity in kinds of tumors, including NSCLC. For example, Liu et al. found that miR133b overexpression sensitized radioresistant lung cancer cells by inhibiting pyruvate kinase isoform M2 (PKM2)-mediated glycolysis [28]. Liu et al. reported that miR-21 downregulation inhibited cell growth, metastasis and reversed chemo- and radio-sensitivity of NSCLC cells by targeting tumor suppressor PTEN [29]. Fan et al. showed that overexpression of miR-15a/16 resulted in an enhancement of radiosensitivity of NSCLC cells by regulating the TLR1/NF- $\kappa B$ signaling pathway [30]. miR-195 was previously confirmed to be significantly reduced and serve as a tumor suppressor in NSCLC cells [31]. Our present studies uncovered that miR195 was down-regulated in NSCLC tissues and cells. Additionally, the expression level of miR195 was significantly reduced after exposing to irradiation. Furthermore, overexpression of miR-195 led to a significant decrease of cell proliferation and colony formation rates, and an obvious increase of apoptosis under irradiation, suggesting that miR-195 overexpression improved radiosensitivity of NSCLC.

Like miRNAs, there are also several reports about the effect of IncRNAs on radiosensitivity of human cancers. For example, Yang et al. implied that knockdown of lncRNA HOX transcript antisense intergenic RNA (HOTAIR) contributed to tumorigenesis and enhanced radiosensitivity of CRC [32]. Lu et al. exhibited that high expression of IncRNA nuclear paraspeckle assembly transcript 1 (NEAT1) regulated radioresistance through modulating mesenchymal transition (EMT) [33]. Lu et al. presented that IncRNA metastasis-associated lung adenocarcinoma transcript 1 (MALAT1) regulated radiosensitivity in HR-HPV-positive cervical cancer by sponging miR-145 [34]. However, no studies about the effect of PVT1 on radiosensitivity of NSCLC cells have been reported.

Previous studies have reported that PVT1 was significantly upregulated in NSCLC and promoted cell proliferation, migration and invasion, and inhibited apoptosis of NSCLC cells $[15,35]$. Consistently, the present study confirmed the abnormal high expression of PVT1 in NSCLC tissues and cells. Notably, we found that irradiation dramatically improved the expression levels of PVT1, and the expressions of PVT1 and miR-195 varied inversely under irradiation. More importantly, PVT1 knockdown remarkably inhibited cell proliferation and colony formation abilities and induced apoptosis under irradiation, indicating that PVT1 knockdown enhanced radiosensitivity of NSCLC. In addition, it is increasingly understood that IncRNAs function as a molecular sponge to competitively inhibit miRNA [25]. Therefore, luciferase reporter assay further revealed that PVT1 could directly interact with miR-195 and negatively regulate its expression. Furthermore, rescue experiments disclosed that miR195 inhibitor completely reversed the inhibition of cell proliferation and colony formation abilities, as well as promotion of apoptosis in vitro mediated by PVT1 knockdown. Also, tumor xenografts experiments suggested that PVT1 knockdown sensitized NSCLC cells to radiotherapy in vivo, which was significantly relieved by miR-195 inhibitor. In line with our study, PVT1 was demonstrated to act as a molecular sponge of miR-195 to promote osteosarcoma development [36].

\section{Conclusion}

Taken together, our study first investigated the effect of PVT1 and its underlying mechanism in regulating radiosensitivity of NSCLC cells. The results indicated that PVT1 knockdown enhanced radiosensitivity of NSCLC cells partly through inhibiting cell proliferation and inducing apoptosis by sponging miR-195, providing a novel therapeutic target to improve radiotherapy efficiency for patients with NSCLC.

\section{KARGER}




\section{Cellular Physiology Cell Physiol Biochem 2017;42:2453-2466 \begin{tabular}{l|l} 
and Biochemistry Published 10.1159/000480209 22,2017 & $\begin{array}{l}\text { (c) 2017 The Author(s). Published by S. Karger AG, Basel } \\
\text { www.karger.com/cpb }\end{array}$
\end{tabular}}

Wu et al.: Knockdown of PVT1 Enhances Radiosensitivity in NSCLC

\section{Disclosure Statement}

The authors of this manuscript state that they have no Disclosure Statement to declare

\section{References}

1 Saika K, Machii R: Cancer mortality attributable to tobacco in Asia based on the WHO Global Report. Jpn J Clin Oncol 2012;42:985.

2 Chen W, Zheng R, Zeng H, Zhang S: The updated incidences and mortalities of major cancers in China, 2011. Chin J Cancer 2015;34:502-507.

- 3 Taylor MD, Nagji AS, Bhamidipati CM, Theodosakis N, Kozower BD, Lau CL, Jones DR: Tumor recurrence after complete resection for non-small cell lung cancer. Ann Thorac Surg 2012;93:1813-1821.

4 Smith RA, Manassaram-Baptiste D, Brooks D, Cokkinides V, Doroshenk M, Saslow D, Wender RC, Brawley OW: Cancer screening in the United States, 2014: a review of current American Cancer Society guidelines and current issues in cancer screening. CA Cancer J Clin 2014;64:30-51.

5 Yang J, Liu H, Wang H, Sun Y: Down-regulation of microRNA-181b is a potential prognostic marker of nonsmall cell lung cancer. Pathol Res Pract 2013;209:490-494.

6 Willers H, Azzoli CG, Santivasi WL, Xia F: Basic mechanisms of therapeutic resistance to radiation and chemotherapy in lung cancer. Cancer J 2013;19:200-207.

7 Raungrut P, Wongkotsila A, Lirdprapamongkol K, Svasti J, Geater SL, Phukaoloun M, Suwiwat S, Thongsuksai P: Prognostic significance of 14-3-3gamma overexpression in advanced non-small cell lung cancer. Asian Pac J Cancer Prev 2014;15:3513-3518.

8 Wang Y, Li Y, Yang Z, Wang D: Genome-wide microarray analysis of long non-coding RNAs in eutopic secretory endometrium with endometriosis. Cell Physiol Biochem 2015;37:2231-2245.

-9 Luo G, Wang M, Wu X, Tao D, Xiao X, Wang L, Min F, Zeng F, Jiang G: Long non-coding RNA MEG3 inhibits cell proliferation and induces apoptosis in prostate cancer. Cell Physiol Biochem 2015;37:2209-2220.

10 Wu J, Wang D: Long noncoding RNA TCF7 promotes invasiveness and self-renewal of human non-small cell lung cancer cells. Hum Cell 2017;30:23-29.

11 Zhai N, Xia Y, Yin R, Liu J, Gao F: A negative regulation loop of long noncoding RNA HOTAIR and p53 in nonsmall-cell lung cancer. Onco Targets Ther 2016; 9: 5713-5720.

12 Fang J, Sun CC, Gong C : Long noncoding RNA XIST acts as an oncogene in non-small cell lung cancer by epigenetically repressing KLF2 expression. Biochem Biophys Res Commun 2016;478:811-817.

13 Sun C, Li S, Zhang F, Xi Y, Wang L, Bi Y, Li D: Long non-coding RNA NEAT1 promotes non-small cell lung cancer progression through regulation of miR-377-3p-E2F3 pathway. Oncotarget 2016;7:51784-51814.

14 Wang Z, Jin Y, Ren H, Ma X, Wang B, Wang Y: Downregulation of the long non-coding RNA TUSC7 promotes NSCLC cell proliferation and correlates with poor prognosis. Am J Transl Res 2016;8:680-687.

15 Yang YR, Zang SZ, Zhong CL, Li YX, Zhao SS, Feng XJ: Increased expression of the lncRNA PVT1 promotes tumorigenesis in non-small cell lung cancer. Int J Clin Exp Pathol 2014;7:6929-6935.

-16 Ding J, Li D, Gong M, Wang J, Huang X, Wu T, Wang C: Expression and clinical significance of the long noncoding RNA PVT1 in human gastric cancer. Onco Targets Ther 2014;7:1625-1630.

-17 Huang C, Yu W, Wang Q Cui H, Wang Y, Zhang L, Han F, Huang T: Increased expression of the lncRNA PVT1 is associated with poor prognosis in pancreatic cancer patients. Minerva Med 2015;106:143-149.

18 Ding C, Yang Z, Lv Z, Du C, Xiao H, Peng C, Cheng S, Xie H, Zhou L, Wu J, Zheng S: Long non-coding RNA PVT1 is associated with tumor progression and predicts recurrence in hepatocellular carcinoma patients. Oncol Lett 2015;9:955-963.

19 Takahashi Y, Sawada G, Kurashige J, Uchi R, Matsumura T, Ueo H, Takano Y, Eguchi H, Sudo T, Sugimachi K, Yamamoto H, Doki Y, Mori M, Mimori K: Amplification of PVT-1 is involved in poor prognosis via apoptosis inhibition in colorectal cancers. Br J Cancer 2014;110:164-171.

-20 Kopp KL, Ralfkiaer U, Nielsen BS, Gniadecki R, Woetmann A, Odum N, Ralfkiaer E: Expression of miR-155 and miR-126 in situ in cutaneous T-cell lymphoma. APMIS 2013;121:1020-1024.

21 Balca-Silva J, Sousa Neves S, Goncalves AC, Abrantes AM, Casalta-Lopes J, Botelho MF, Sarmento-Ribeiro AB, Silva HC: Effect of miR-34b overexpression on the radiosensitivity of non-small cell lung cancer cell lines. Anticancer Res 2012;32:1603-1609. 


\section{Cellular Physiology Cell Physiol Biochem 2017;42:2453-2466 \begin{tabular}{l|l} 
DOI: 10.1159/000480209 22,2017 & $\begin{array}{l}\text { O 2017 The Author(s). Published by S. Karger AG, Basel } \\
\text { www.karger.com/cpb }\end{array}$ \\
and Biochemistry Publisned online: August 22,
\end{tabular}}

Wu et al.: Knockdown of PVT1 Enhances Radiosensitivity in NSCLC

-22 Liu B, Qu J, Xu F, Guo Y, Wang Y, Yu H, Qian B: MiR-195 suppresses non-small cell lung cancer by targeting CHEK1. Oncotarget 2015;6:9445-9456.

-23 Yongchun Z, Linwei T, Xicai W, Lianhua Y, Guangqiang Z, Ming Y, Guanjian L, Yujie L, Yunchao H: MicroRNA-195 inhibits non-small cell lung cancer cell proliferation, migration and invasion by targeting MYB. Cancer Lett 2014;347:65-74.

24 Guo H, Li W, Zheng T, Liu Z: MiR-195 targets HDGF to inhibit proliferation and invasion of NSCLC cells. Tumor Biol 2014;35:8861-8866.

25 Li C, Chen J, Zhang K, Chen L: Progress and prospects of long noncoding RNAs (lncRNAs) in hepatocellular carcinoma. Cell Physiol Biochem 2015;36:423-434.

-26 Laine AM, Westover KD, Choy H: Radiation therapy as a backbone of treatment of locally advanced nonsmall cell lung cancer. Semin Oncol 2014;41:57-68.

27 Kang J, Kim E, Kim W, Seong KM, Youn H, Kim JW, Kim J, Youn B: Rhamnetin and cirsiliol induce radiosensitization and inhibition of epithelial-mesenchymal transition (EMT) by miR-34a-mediated suppression of Notch-1 expression in non-small cell lung cancer cell lines. J Biol Chem 2013;288:2734327357.

28 Liu G, Li YI, Gao X: Overexpression of microRNA-133b sensitizes non-small cell lung cancer cells to irradiation through the inhibition of glycolysis. Oncol Lett 2016;11:2903-2908.

29 Liu ZL, Wang H, Liu J, Wang ZX: MicroRNA-21 (miR-21) expression promotes growth, metastasis, and chemo- or radioresistance in non-small cell lung cancer cells by targeting PTEN. Mol Cell Biochem 2013;372: 35-45.

-30 Lan F, Yue X, Ren G, Li H, Ping L, Wang Y, Xia T: miR-15a/16 enhances radiation sensitivity of non-small cell lung cancer cells by targeting the TLR1/NF-kappaB signaling pathway. Int J Radiat Oncol Biol Phys 2015;91:73-81.

-31 Wang X, Wang Y, Lan H, Li J: MiR-195 inhibits the growth and metastasis of NSCLC cells by targeting IGF1R. Tumour Biol 2014;35:8765-8770.

-32 Yang XD, Xu HT, Xu XH, Ru G, Liu W, Zhu JJ, Wu YY, Zhao K, Wu Y, Xing CG, Zhang SY, Cao JP, Li M: Knockdown of long non-coding RNA HOTAIR inhibits proliferation and invasiveness and improves radiosensitivity in colorectal cancer. Oncol Rep 2016;35:479-487.

-33 Lu Y, Li T, Wei G, Liu L, Chen Q, Xu L, Zhang K, Zeng D, Liao R: The long non-coding RNA NEAT1 regulates epithelial to mesenchymal transition and radioresistance in through miR-204/ZEB1 axis in nasopharyngeal carcinoma. Tumour Biol 2016;37:11733-11741.

-34 Lu H, He Y, Lin L, Qi Z, Ma L, Li L, Su Y: Long non-coding RNA MALAT1 modulates radiosensitivity of HRHPV+ cervical cancer via sponging miR-145. Tumour Biol 2016;37:1683-1691.

-35 Wan L, Sun M, Liu GJ, Wei CC, Zhang EB, Kong R, Xu TP, Huang MD, Wang ZX: Long noncoding RNA PVT1 promotes non-small cell lung cancer cell proliferation through epigenetically regulating LATS2 expression. Mol Cancer Ther 2016;15:1082-1094.

-36 Zhou Q Chen F, Zhao J, Li B, Liang Y, Pan W, Zhang S, Wang X, Zheng D: Long non-coding RNA PVT1 promotes osteosarcoma development by acting as a molecular sponge to regulate miR-195. Oncotarget 2016;7:82620-82633. 